\title{
Aprendizagem Baseada em Problemas no Currículo Integrado Para Graduação em Medicina:
} Construção Da Autonomia Acadêmica

\author{
Taylor Tito Bobato ${ }^{1}$ \\ Izabel Cristina Meister Martins Coelho² \\ Elaine Rossi Ribeiro ${ }^{3}$
}

${ }^{1}$ Docente do Curso de Fisioterapia da Faculdade Evangélica do Paraná (Fepar) e nos cursos de Enfermagem, Biomedicina, Farmácia e Medicina da Associação Hospitalar de Proteção à Infância Dr. Raul Carneiro. Fisioterapeuta do Instituo Terapêutico Curitiba e da Prefeitura Municipal de Contenda, Paraná. Tem experiência nas áreas de Traumato-Ortopedia Funcional, Terapia Manual, Osteopatia, Reeducação postural Global (RPG). Mestre em Ensino nas Ciências da Saúde (FPP). Orcid: <http://orcid.org/00000002-3356-9397>.flexor@terra.com.br

2 Médica pela Universidade Federal do Paraná (1989), com Residência Médica em Cirurgia Geral pela Universidade Federal do Paraná (1992) e Cirurgia Pediátrica no Complexo Hospitalar Pequeno Príncipe (1995). Doutora (2003) e mestre em Clínica Cirúrgica pela Universidade Federal do Paraná (1999), recebendo com sua tese prêmio de pesquisa pela American Society of Parenteral and Enteral Nutrition (Aspen) em 2004. Observership no Serviço de Transplante Multivisceral e Hepático, Division of Transplantation, University of Miami (2001). Especialista em Ativação de Processos de Mudança na Formação Superior de Profissionais de Saúde promovido pelo MS/Fiocruz/Rede Unida (2006) e em Educação para Profissionais da Área de Saúde pela UFC/Faimer BRASIL (2010). Professora do curso de Medicina desde 1998, coordenadora do Internato de Medicina (2004-2005) e coordenadora do curso de Medicina da Faculdade Evangélica do Paraná (2005-2009) em Curitiba. Professora convidada da ENSP/Fiocruz, atuando como Tutora na Formação Superior de Profissionais de Saúde (2006/2011). Em 2010/11 atuou como diretora técnica do Hospital Municipal, diretora de Educação em Saúde e secretária municipal de Saúde de São José dos Pinhais. Em 2011 iniciou a coordenação do projeto Pró-Ensino na Saúde no Programa de Pós-Graduação stricto sensu das Faculdades Pequeno Príncipe. É cirurgiã da Equipe de Transplante Hepático do Complexo Pequeno Príncipe (desde 2000) e do Hospital de Clínicas da Universidade Federal do Paraná (desde 2004). Atualmente coordena o curso de Medicina e o Programa de Mestrado Acadêmico em Ensino nas Ciências da Saúde nas Faculdades Pequeno Príncipe. É membro da Camem - MEC. Orcid: <http://orcid.org/0000-0002-4904-0746>. izabel.coelho@fpp.com.br

3 Possui Graduação em Enfermagem pela Universidade Estadual de Londrina (1978), Mestrado em Educação pela Universidade Estadual de Londrina (1998), Mestrado em Medicina (Clínica Cirúrgica) pela Universidade Federal do Paraná (2004) e Doutorado em Medicina (Clínica Cirúrgica) pela Universidade Federal do Paraná (2006). Diretora acadêmica da Faculdade Evangélica do Paraná por 6 anos. Exerceu a superintendência técnica do Instituto Municipal de Administração Pública de Curitiba - Imap, tendo sido Diretora da Escola de Administração Pública da Prefeitura de Curitiba. Docente de cursos de Pós-Graduação nas áreas de planejamento, gestão e liderança. Tem experiência na área de Gestão e Educação, com ênfase em liderança e avaliação. Atualmente é diretora acadêmica do Instituto de Ensino e Pesquisa - IEP/Feaes e pesquisadora docente da Faculdade Pequeno Príncipe em Curitiba e do Programa de Pós-Graduação stricto sensu Ensino na Ciência da Saúde. Participante do Grupo de Pesquisa Pensa. Orcid: <http://orcid.org/0000-0003-3492-217X>. elaine.rossi@ hotmail.com 


\title{
Resumo
}

Este artigo tem como objetivo analisar como o estudante de Medicina constrói a sua autonomia acadêmica em um currículo integrado baseado especialmente na Aprendizagem Baseada em Problemas (APB) ou PBL (Problem Based Learning). Foi realizada uma pesquisa exploratória descritiva, de cunho quantitativo, transversal, com alunos do curso de Medicina de uma Instituição de Ensino Superior (IES) no município de Curitiba. Para a coleta das informações foi aplicado um questionário com 35 afirmações utilizando-se escala Likert de 5 pontos. Nesta análise o perfil dos estudantes mostrou que a média de idade nas turmas avaliadas esteve entre 19 e 21 anos, que houve predominância do gênero feminino, que grande parte dos estudantes mora com familiares e que a maioria não trabalha e não tem outra formação. Praticamente a totalidade dos estudantes não teve contato com o método antes de ingressar no curso de Medicina e, após este contato, seu conhecimento sobre 0 método, a compreensão do desenvolvimento do tutorial e a integração teoria/prática ocorreu de forma imediata. Observou-se que, à medida que passam os semestres, há consciência sobre a importância da autoavaliação e da capacidade de inter-relação e cooperação de trabalho em grupo, o que exercita sua habilidade de resolução de problemas e desenvolve o pensamento científico nas habilidades médicas futuras, posto que a estatística descritiva comprovou resultados significativos na construção da autonomia destes estudantes.

Palavras-chave: Aprendizagem baseada em problemas. Autonomia. Medicina. Currículo.

\section{PROBLEM BASEAD LEARNING IN THE INTEGRATED CURRICULUM FOR GRADUATION IN MEDICINE: CONSTRUCTION OF ACADEMIC AUTONOMY}

\begin{abstract}
This article aims to analyze the initial vision of the student, to understand how their adaptation occurs, to list the factors of success in the adaptation and their difficulties to the method in an integrated curriculum. An exploratory descriptive, quantitative, cross-sectional research was carried out with students of the medical course of a Higher Education Institution (IES) in the Curitiba city. For the collection of information, a questionnaire with thirty-five (35) affirmations was applied on a Likert scale with five points. In this analysis the profile of the students evaluated showed that the average age in the classes evaluated was between 19 and 21 years, there was predominance of the female gender, most of the students live with relatives, the majority do not work and have no other training. Practically all students had no contact with the method before entering in medical school and after this contact, their knowledge about the method, the understanding of the tutorial development and integration theory / practice occurred immediately. It has been observed that, as the semesters pass, there is awareness of the self-assessment importance, interrelationship capacity and group work cooperation, which exercises their problem-solving ability and develops scientific thinking in future medical skills and the descriptive statistics showed significant results in the construction of the autonomy of these students.
\end{abstract}

Keywords: PBL (Problem Based Learning). Autonomy. Medicine. Integrated curriculum.

Recebido em: 23/5/2017

Aceito em: 22/11/2017 
Desde a segunda metade do século 20, em diversos países, a formação dos profissionais da área da saúde é tema frequente nas discussões educacionais. Na esfera da educação médica, destacam-se as críticas aos métodos tradicionais de ensino, os quais não mais atendem ao perfil de formação dos profissionais que a atual sociedade demanda, ou seja, médicos com maiores possibilidades de agir na resolução dos problemas de saúde dos indivíduos e da coletividade, mais comprometidos com uma postura simultaneamente técnica, ética e política, transformando os processos fragmentados de trabalho em ações de cuidado (SMOLKA, 2011).

Novos desafios se impõem nos cenários atuais de educação e currículos universitários altamente complexos nas escolas médicas. O acúmulo exponencial de conhecimentos e a incorporação de novas tecnologias impulsionam para uma formação médica fragmentada em campos altamente especializados e busca pela eficiência técnica. Na área da saúde, este cenário vem ganhando contornos próprios, à medida que se constata a indissociabilidade entre teoria e prática, na perspectiva da visão holística na formação do homem, bem como na "ampliação da concepção de cuidados, que se tornou essencial para o adequado desempenho laboral" (SOUZA; IGLESIAS; PAZIN FILHO, 2014, p. 47).

É sabido que currículos inovadores tendem a priorizar métodos ativos de ensino-aprendizagem, provocando mudanças no processo de educação, definindo, por sua vez, uma aprendizagem baseada em resultados e competências, favorecendo o desenvolvimento de novas atitudes nos estudantes. $\mathrm{O}$ ensino e aprendizagem dos conhecimentos elaborados pela ciência, pela filosofia e pelas artes, são recursos fundamentais para a ampliação de uma consciência mais ampla principalmente nas metodologias ativas (LUCKESI, 2011, p. 55).

As metodologias ativas ajudam os estudantes na aquisição de conhecimentos teóricos, com base na observação da realidade e na reflexão crítica sobre as ações dos sujeitos, de forma que os conteúdos teóricos são apreendidos em conexão com a prática. Os estudantes que participam de metodologias ativas de aprendizagem exercitam transformar os impasses e as impossibilidades em trabalhos e ações possíveis. Essas metodologias também contribuem para mu- 
danças no modo de pensar e agir dos profissionais de saúde, os quais passaram a interagir em um espaço plural de interesses, potencialidades e capacidades (FREITAS; RODRIGUES, 2005).

Diante deste cenário surgem, então, propostas metodológicas de ensino-aprendizagem, entre elas o Problem Based Learning (PBL) (SMOLKA, 2011). Esta metodologia consiste numa concepção pedagógica que ultrapassa a forma tradicional de ensinar e aprender. Tem por característica ser centrada no estudante, proporcionando um ambiente propício ao desenvolvimento da capacidade para construir ativamente a própria aprendizagem, por meio de problemas selecionados para o estudo, desenvolver e utilizar o raciocínio crítico e as habilidades de comunicação para a resolução de problemas clínicos, entendendo a necessidade de aprender ao longo da vida. Diferencia-se, portanto, do ensino tradicional, no qual, muitas vezes, o ensinar é meramente uma transmissão de informações (BRANSFORD; BROWN; COCKING, 2007).

Na década de 70 do século 20, o PBL foi introduzido na Universidade de Maastricht, na Holanda, e em Newcastle, na Austrália. Seguiu-se a este pioneirismo a inserção do PBL na Escola de Medicina de Harvard, em 1984, e em outras universidades estadunidenses, canadenses e europeias (RIBEIRO, 2005).

No Brasil, duas escolas médicas foram as pioneiras na introdução do currículo APB ou PBL: a Faculdade de Medicina de Marília (Famema), em São Paulo, e o curso de Medicina da Universidade Estadual de Londrina (UEL), no Paraná, em 1997 e 1998, respectivamente.

Claramente, a educação de profissionais de saúde tem concentrado esforços na discussão dos conteúdos ou saberes necessários à formação de profissionais generalistas ou que atendam às reais necessidades da população. Tratar dos processos de religação e reconstrução dos saberes é absolutamente vital para que as propostas de mudança alcancem a necessária relevância social (MORIN, 2002).

Os estudantes chegam à universidade com uma série de conhecimentos, habilidades, crenças e conceitos prévios que, em certa medida, podem influenciar sua capacidade de raciocínio, de solução de problemas e aquisição de novos conhecimentos, o que nos faz refletir sobre o currículo que se tem prescrito para 
a formação de profissionais de saúde. Piaget argumenta que a autonomia não se desenvolve em uma atmosfera de autoridade, opressão intelectual e moral. Ao contrário, para que ela ocorra, são fundamentais as vivências em situações de cooperação, liberdade de pesquisa, respeito mútuo e também a experiência de vida. Sob a perspectiva da autonomia, os conflitos são necessários ao desenvolvimento do estudante, devendo ser encarados como ricas oportunidades de se trabalhar valores e regras (MALCON, 2012).

Dentro de um currículo que tem como base a utilização de metodologias ativas, o papel do educador não é priorizar a solução do conflito em si, mas o processo de resolução e a forma com que os envolvidos possam enfrentar o problema, aprender com o ocorrido, estimulando o desenvolvimento de sua autonomia.

Assim, foi elaborada uma proposta de investigação que respondesse à seguinte questão: Como o estudante de Medicina constrói a sua autonomia acadêmica em um currículo baseado nas Metodologias Ativas de Ensino-Aprendizagem (Maea), especialmente na Aprendizagem Baseada em Problemas?

Para responder a esta questão estabeleceu-se como objetivo analisar como o estudante de Medicina constrói a sua autonomia acadêmica em um currículo integrado baseado especialmente no PBL (Problem Based Learning) ou APB.

\section{Revisão de Literatura}

Na perspectiva construtivista de Piaget, independentemente do estágio em que o ser humano se encontra, a aquisição de conhecimento é ação do sujeito sobre o objeto, ou seja, o conhecimento humano se constrói na interação homem-meio, sujeito-objeto. O processo começa com o entendimento do elemento novo, com a incorporação das estruturas já esquematizadas mediante a interação. Há mudanças no sujeito e tem início o processo de acomodação, que aos poucos chega à organização interna, começa a adaptação externa do sujeito e a internalização já acontece; um novo desequilíbrio volta a acontecer e pode ser provocado por carência, curiosidade, dúvida, entre outros (MENDES et al., 2012). 
A ciência destaca a importância de o estudante assumir o controle de sua própria aprendizagem, considerando que deve identificar quando entende e quando precisa de mais informações, e que estratégias pode utilizar para avaliar as suas próprias teorias sobre os novos conhecimentos (ENILTON, 2012).

A aprendizagem ativa tem como base a cognição e a metacognição. Cognição refere-se a um tipo específico de representação dos objetos e fatos (isto é, representações proposicionais) e, num sentido lato, a qualquer tipo de representação da informação proveniente do meio, incluindo todos os tipos de representações multidimensionais. Já a metacognição é a capacidade de uma pessoa de prever o próprio desempenho em diversas tarefas e de monitorar seus níveis atuais de domínio e compreensão, conhecimento do próprio conhecimento, avaliação, regulação, organização dos processos cognitivos (RIBEIRO, 2003).

Esta nova ciência de aprendizagem enfatiza a importância de repensar o que é ensinado, a maneira de ensinar e o modo de avaliar esta aprendizagem, tendo sempre como foco o estudante, estimulando-os a serem ativos, assumindo sua própria aprendizagem por meio da definição de objetivos de aprendizagem e do monitoramento de seu progresso em alcançá-los, necessitando, para tanto, autonomia no seu processo de aprendizagem (BASTOS, 2006).

O PBL trouxe respostas positivas ao processo de aprendizagem, apresentando como um dos fundamentos principais a proposta de ensinar o estudante a partir do seu próprio aprendizado, permitindo que busque o conhecimento nos inúmeros meios de difusão disponíveis, deixando de ser centrada no professor, possibilitando que o estudante aprenda a utilizar e a pesquisar estes meios mediante um currículo integrador que sofre retroalimentações constantes, objetivando atender às exigências do corpo discente, na efetivação do seu processo de autonomia na construção do conhecimento (RIBEIRO, 2005).

O conhecimento inicia-se por um problema e se encerra com a sua resolução, passando assim por um processo indagativo, reflexivo e ordenado de ideias. Todo o ato de pensar é original e consequentemente favorecerá a descoberta, possibilitando a criação do prazer da produtividade intelectual e não só do armazenamento de informações 
transmitidas por terceiros. Nesse sentido, fazem-se necessárias condições que estimulem o pensamento e a autonomia para que o aprendizado se desenvolva (BASTOS, 2006).

Todo este processo indagativo e reflexivo predispõe o adulto a aprender aquilo que deve saber e precisa para se tornar capacitado a enfrentar as situações da vida real. Uma fonte particularmente rica de "prontidão para aprender" são as tarefas associadas à passagem de um estágio de desenvolvimento para o próximo. As experiências de aprendizagem devem estar sincronizadas com essas tarefas de desenvolvimento. A prontidão pode ser induzida por meio da exposição a exercícios de simulação e outras técnicas (KNOWLES; HOLTON; SWANSON, 2009).

O desenvolvimento da metodologia da Aprendizagem Baseada em Problema-valoriza, questiona e contextualiza a capacidade de pensar dos estudantes numa forma gradativa e autônoma de aquisição de um conhecimento relativo para resolver situações reais em projetos referentes aos conteúdos na área de estudos, que tem como meta o desenvolvimento no aspecto físico, emocional e intelectual, por meio de métodos experimentais que estimulam a construção individual do próprio conhecimento e fortalecendo o já existente (GRANT, 2002).

A palavra “autonomia", no Dicionário Aurélio (BUARQUE, 2010), tem os seguintes significados: faculdade de se governar por si mesmo, direito ou faculdade que tem uma nação de se reger por leis próprias. Autônomo é aquele não depende dos outros, que tem ou em que há autonomia. Concorrem para a promoção da autonomia as atividades de aprendizagem que possibilitam, em relação a um dado comportamento, um envolvimento pessoal e alta flexibilidade em sua execução, bem como percepção de liberdade psicológica e de escolha. No ambiente escolar tradicional, a competição e as notas são percebidas como poderosas fontes de controle, interferindo na promoção da autonomia do estudante, como estratégia motivacional na escola.

O estabelecimento de metodologias ativas favorece a motivação autônoma, quando inclui o fortalecimento da percepção do estudante de ser origem da própria ação, ao serem apresentadas oportunidades de problematização de situações envolvidas na programação escolar, de escolha de aspectos dos conte- 
údos de estudo, de caminhos possíveis para o desenvolvimento de respostas ou soluções para os problemas que se apresentam como alternativas criativas para a conclusão do estudo ou da pesquisa, entre outras possibilidades (KNOWLES; HOLTON; SWANSON, 2009).

O PBL pode ser visto como um exemplo de ambiente, que promove a aprendizagem ativa, construtiva, contextual, cooperativa e de aprendizagem dirigida por objetivos. É um processo que estimula a autoaprendizagem e facilita a educação continuada porque desperta a curiosidade do estudante e, ao mesmo tempo, oferece meios para que possa desenvolver a capacidade de análise de situações, com ênfase nas condições locais regionais, e apresentar soluções em consonância com o perfil psicossocial da comunidade na qual está inserido (GOMES, 2010).

A Aprendizagem Baseada em Problemas consiste numa concepção pedagógica que ultrapassa a forma tradicional de ensinar e aprender, caracterizando-se principalmente por colocar o estudante no centro do processo, criando um ambiente propício ao desenvolvimento da capacidade de: (1) construir ativamente a própria aprendizagem; (2) articular os conhecimentos prévios com o estímulo proporcionado pelos problemas selecionados para o estudo; (3) desenvolver e utilizar o raciocínio crítico e as habilidades de comunicação para a resolução de problemas clínicos, e entender a necessidade de aprender ao longo da vida, diferenciando-se do ensino tradicional, no qual, muitas vezes, o ensinar é meramente uma transmissão de informações ao estudante (BERBEL, 1996).

\section{Metodologia}

\section{Tipo de Estudo}

Realizou-se uma pesquisa exploratória descritiva, de cunho quantitativo e transversal. O estudo teve como cenário uma Instituição de Ensino Superior (IES) privada de caráter filantrópico no município de Curitiba. A IES foi credenciada pelo MEC há 11 anos, trabalha exclusivamente com cursos na área de saúde, como Enfermagem, Farmácia, Biomedicina, Psicologia e Medicina. 
Aplicou-se um questionário aos estudantes de curso de Graduação em Medicina, com perguntas referentes aos dados sociodemográficos e, na sequência, 35 afirmativas relacionadas ao próprio desempenho; à compreensão do seu papel no método; à participação efetiva nas diferentes atividades; à percepção de sua evolução acadêmica por meio da construção do conhecimento. Para as respostas, utilizou-se cinco itens da Escala de Likert. Ressalta-se que o instrumento foi validado por 12 especialistas selecionados de acordo com a sua formação em PBL e currículo integrado.

Sequencialmente foram selecionados estudantes de todos os períodos do ano de 2016 do curso de Medicina, maiores de 18 anos, que aceitaram participar da pesquisa e assinaram o Termo de Consentimento Livre e Esclarecido (TCLE) e preencheram completamente o Instrumento de Pesquisa (IP), o qual foi desenhado com nove domínios, a saber: D1: aprendizado e autocrítica; D2: intencionalidade do método: compreensão do tutorial; D3: autoavaliação; D4: estudo dirigido; D5: papel do médico; D6: profissionalismo; D7: integração teórico-prática; D8: conhecimento do método e D9: autonomia na resolução de problemas. Para cada domínio uma série de afirmativas foi apresentada aos estudantes, totalizando 35 afirmações. Foram excluídos deste estudo menores de 18 anos e aqueles que não preencheram o IP de acordo. A organização ocorreu da seguinte forma: estudantes do primeiro período identificados como turma A, do segundo período identificado como turma $\mathrm{B}$, do terceiro período identificado como turma $\mathrm{C}$, do quarto período identificado como turma $\mathrm{D}$ e os egressos do vestibular de inverno (segundo semestre) identificados como turma E.

Para responder à questão de pesquisa, este trabalho foi dividido em duas fases. Na primeira fase foram aplicados o Termo de Consentimento Livre e Esclarecido (TCLE) e o Instrumento de Pesquisa aos estudantes da turma A ( $1^{\circ}$ período), turma $\mathrm{B}\left(2^{\circ}\right.$ período), turma $\mathrm{C}\left(3^{\circ}\right.$ primeiro) e turma $\mathrm{D}\left(4^{\circ}\right.$ período), levando-se em consideração a seguinte ordem: na turma A estudantes preencheram o IP no início do semestre, com o objetivo de obter um impacto inicial desta turma. As demais turmas $(\mathrm{B}, \mathrm{C}$ e $\mathrm{D})$ realizaram o preenchimento do IP no final do semestre (considerando maior compreensão do método) todos no primeiro semestre de 2016. 
$\mathrm{Na}$ segunda fase foi aplicado novamente o IP respeitando a mesma ordem da primeira fase, só que agora sendo aplicado a todas as turmas ao final do segundo semestre de 2016. Neste semestre a turma A passa ser o segundo período, turma $\mathrm{B}$ o terceiro período, turma $\mathrm{C}$ o quarto período e turma $\mathrm{D}$ o quinto período. Também ao final do primeiro módulo foi aplicado o IP para a turma nova $\mathrm{E}$ (primeiro período), que ingressou no segundo semestre (vestibular de inverno), este sendo aplicado apenas uma vez, para parear com as respostas iniciais da turma A.

$\mathrm{Na}$ análise dos dados e validação do instrumento de pesquisa pelo teste de confiabilidade, o critério empregado foi o coeficiente alfa de Cronbach, que visa a estimar a fidelidade do constructo. $\mathrm{O}$ valor mínimo aceitável para o alfa está entre 0,6 e 0,7 (HAIR et al., 2005).

A seguir foi realizada a análise que permitiu mensurar objetivamente a construção da autonomia do estudante de Medicina quanto a sua inserção neste método de aprendizagem ativa, o PBL. Quanto à análise de dados, inicialmente as variáveis foram submetidas a estatísticas descritivas (frequências absolutas e frequências relativas percentuais). Em seguida, os dados quantitativos foram avaliados por meio do teste de Qui-Quadrado para Independência, avaliando as variáveis de respostas em razão de diferentes características dos estudantes do curso de Medicina. Em caso de significância estatística no teste de Qui-Quadrado foi aplicado o teste de acompanhamento de Resíduos Ajustados.

\section{Resultados e Discussões}

Foram coletados e analisados dados de 152 estudantes (em um universo de 200) como: idade, gênero, formação, com quem mora, se trabalha, período do curso, se possui outro curso superior, como ingressou no curso e estado civil. Nesta análise observou-se que a média de idade nas quatro turmas avaliadas esteve entre 19 e 21 anos, e também há predominância do gênero feminino, chegando a $68 \%$ de todas as turmas. Uma grande parte dos estudantes mora com familiares, chegando a um percentual de $81,8 \%$ na turma B. Em relação ao trabalho, $97 \%$ não exerce outra atividade, chegando a 100\% nas turmas $\mathrm{A}$ 
e C. O mesmo ocorre no item "outra formação", obtendo $100 \%$ de resposta "não" na turma C. A maior parte dos estudantes é solteira, alcançando 100\% nas turmas $\mathrm{C}$ e D.

Após análise do perfil sociodemográfico dos estudantes, uma análise fatorial exploratória foi realizada com o intuito de definir variáveis latentes a partir de indicadores do instrumento de avaliação construído. Uma variável latente é um conjunto de indicadores que expressa uma dada característica impossível de ser avaliada sob um único aspecto, resumindo os dados em um número menor de conceitos, também chamados domínios, simplificando assim a interpretação dos resultados. Esses domínios são conjuntos de indicadores que compõem um grupo de questões que apresentam maior correlação no entendimento de suas composições e características. Para definir quais indicadores compõem cada domínio, foram calculadas as cargas fatoriais entre os indicadores e os domínios. Por fim os domínios foram nomeados com base nos indicadores que os compõem, com o intuito de agregar significado a eles. Os indicadores com cargas fatoriais altas foram considerados os mais importantes, o que permitiu atribuir-se um nome para cada domínio. Segundo a estatística, foi possível nomear os domínios tal como segue: domínio 1 (D1) Aprendizado e Autocrítica; o domínio 2 (D2) Intencionalidade do Método; o domínio 3 (D3) Autoavaliação; o domínio 4 (D4) Estudo Dirigido; o domínio 5 (D5) Papel do Médico; D6: Profissionalismo; D7: Integração Teórico-Prática; D8: Conhecimento do Método; D9: Autonomia na Resolução de Problemas.

Considerando a finalidade de atender ao objetivo proposto, definiu-se que os domínios aderentes ao desenvolvimento da autonomia são: aprendizado e autocrítica, auto- avaliação, estudo dirigido e autonomia na resolução de problemas.

No domínio1, aprendizado - autocrítica, os indicadores dizem respeito à resolução de problemas, pensamento autocrítico, envolvimento no seu próprio processo de aprendizagem, compreensão da metodologia e se o momento tutorial favorece um raciocínio clínico e melhor fixação da teoria. 
Foi possível observar que não houve diferença entre as médias das turmas entre o $1^{\circ}$ e $2^{\circ}$ semestre $\left(F_{1}=2,75 ; p\right.$-valor $\left.=0,10\right)$, indicando que entre um semestre e outro os indivíduos mantiveram a mesma percepção sobre o domínio em questão, contudo entre as turmas avaliadas houve diferença estatística significativa $\left(\mathrm{F}_{2}=52,12 ; \mathrm{p}\right.$-valor $\left.<0,001\right)$, sendo possível observar que a turma $\mathrm{D}$ apresentou as maiores médias de respostas para D1, seguido pelas médias das turmas $\mathrm{B}, \mathrm{A}$ e $\mathrm{C}$, respectivamente.

A interação entre o tempo e as turmas também foi significativa $\left(\mathrm{F}_{1,4}=76,91\right.$; p-valor $<0,001$ ), com aumento da média de respostas nas turmas A, B e C, entre o primeiro e o segundo semestre, e uma queda nas médias na turma $\mathrm{D}$.

A maior média de resposta da turma $\mathrm{D}$ poderia ser justificada por ser o período mais avançado do curso que vivenciou o PBL com mais tempo. Assim teriam desenvolvido retroalimentação de conhecimentos e consequente metacognição, o mesmo ocorrendo em ordem decrescente nas turmas B e A.

De forma inesperada a turma $\mathrm{C}\left(3^{\circ}\right.$ e $4^{\circ}$ períodos $)$ apresenta índices menores neste domínio. Algumas possibilidades em que a literatura pode explicar a situação: ansiedade do estudante, preparo do docente (adaptação/evolução) e fases da instituição do curso. Houve constância no crescimento das outras turmas, quando comparadas a elas mesmas no semestre 1, apesar da menor resposta da turma $\mathrm{C}$.

No domínio 3 (D3), autoavaliação, o estudante faz uma reflexão e autocrítica em relação a sua competência e com seu desempenho. Os alunos mantêm sua média de respostas nas quatro turmas, demonstrando que desde o primeiro semestre eles entendem que neste método a autoavaliação é importante para o autoaprendizado, mantendo-se esta percepção nas quatro turmas.

Para Enilton (2012), a autoavaliação está associada como uma fase do processamento de alto nível, capacidade do indivíduo de monitorar, autorregular e elaborar estratégias para potencializar a cognição.

O domínio 4 (D4), estudo dirigido, foi focado no entendimento sobre se o estudo dirigido permite uma melhor aprendizagem. 
O estudo dirigido estimula a formação de novas conexões e está ligado à metacognição, que tem papel importante nos processos de aprendizagem e autoavaliação, no julgamento da aprendizagem e monitoramento da compreensão.

Segundo Freire (2010), ensinar não é transferir conhecimento, mas criar possibilidades para sua produção e construção, e que os discentes se tornem sujeitos autônomos do próprio pensamento, da própria aprendizagem, saindo do modelo tradicional.

No primeiro semestre de avaliação observam-se maiores médias para D4 nas turmas A, B e D, estando a turma C com a menor média entre as turmas avaliadas.

No segundo semestre de avaliação temos uma queda nas médias das turmas A e B, com aumento da média na turma C, havendo assim uma inversão de valores entre essas turmas. A turma $\mathrm{D}$ permaneceu com uma média de respostas alta em D4, entre o primeiro e o segundo semestre avaliados (turma D).

$\mathrm{O}$ aumento das médias de resposta das turmas $\mathrm{C}$ e $\mathrm{D}$, no segundo semestre pode estar relacionado com o aumento da interação professor/estudante, estudante/estudante, pela experiência adquirida ao longo do tempo.

Os grupos tutoriais são a estratégia central do PBL. Neles são analisados problemas estruturados a partir do currículo, que visam a permitir a discussão contextualizada dos tópicos, favorecendo a recuperação do conhecimento prévio e a aquisição de novos saberes. Além disso, os grupos tutoriais favorecem o desenvolvimento de outras habilidades, como comunicação e trabalho em equipe, solução de problemas e desenvolvimento de postura crítica (TOLEDO; IBIAPINA; LOPES, 2008).

Para o domínio 9 (D9), houve diferença significativa entre as turmas $\left(F_{2}=10,99 ; \mathrm{p}<0,001\right)$ e considerando a interação entre os períodos de avaliação e as turmas $\left(\mathrm{F}_{1,4}=6,96 ; \mathrm{p}<0,001\right)$. Percebe-se que há um aumento da média de respostas para $\mathrm{D} 9$ na turma $\mathrm{A}$, entre o primeiro e o segundo semestres avaliados, e uma queda nas médias de respostas na turma B. 


\section{Conclusão}

Este estudo demonstrou que a quase totalidade dos estudantes não teve contato com o método antes de ingressar no curso de Medicina e que após este contato seu conhecimento sobre o método, a compreensão do desenvolvimento do tutorial e integração teoria/prática ocorreram de forma imediata. Nos domínios que demonstraram a execução do método, D1, D4 e D9, houve compreensão progressiva, significativamente demonstrada.

O desenvolvimento da habilidade de pensamento crítico no domínio (D1) e os passos seguidos durante os tutoriais apresentaram progressão entre as turmas, demonstrando adaptação ao método. Entre outros fatores de sucesso, os módulos de Habilidades Médicas e Integração Ensino-Comunidade (D6 P<0,05) e estudo dirigido (entre turmas e entre semestres) têm papel fundamental, sendo percebidos no aprofundamento dos conhecimentos adquiridos e numa visão clara de suas futuras atividades profissionais, mostrando que a percepção do estudante sobre o método melhora ao longo do curso.

Os estudantes que participaram deste estudo percebem resultados significativos na construção da sua autonomia, pela compreensão dos módulos, pela interação entre eles do primeiro ao oitavo período, construindo uma percepção real sobre sua futura profissão. Outro ponto importante e decisivo ocorre nos momentos tutoriais, quando o aluno tem a oportunidade de compartilhar conhecimentos, sendo estimulado a ter consciência da importância da autoavaliação, de sua capacidade de inter-relação e cooperação de trabalho em grupo, o que exercita sua habilidade de resolução de problemas e desenvolve o pensamento científico nas habilidades médicas futuras e não nas habilidades de memória percebidas no domínio D1, D4, e D9. Conclui-se que a autonomia do estudante é progressiva, sendo evidenciada nos domínios D1(aprendizado teórico-crítico), D4 (estudo dirigido) e D9 (autonomia na resolução de problemas).

A continuidade deste estudo nos períodos seguintes, inclusive no internato, poderá possibilitar uma melhor e profunda compreensão da percepção de como o estudante constrói sua autonomia acadêmica em um currículo baseado nas metodologias ativas de ensino/aprendizagem, especialmente no PBL. 


\section{Referências}

BASTOS, C. C. Metodologias ativas. 2006. Disponível em: <http://educacaoemedicina. blogspot.com.br/2006/02/metodologias-ativas.html>. Acesso em: 13 nov. 2016.

BERBEL, N. A. N. A problematização e a aprendizagem baseada em problemas: diferentes termos ou diferentes caminhos? Interface - Comunicação Saúde Educação, v. 2, n. 2, p. 139-154, 1996.

BRANSFORD, J. D.; BROWN, A. L.; COCKING, R. R. Como as pessoas aprendemcérebro mente experiência e escola. São Paulo: Senac, 2007.

BUARQUE, Aurélio B. Minidicionário Aurélio da Língua Portuguesa. Nova ortografia. 8. Ed. São Paulo: Positivo, 2010.

ENILTON F. R. Os dezpressupostos andragógicos da aprendizagem do adulto: um olhar diferenciado na educação do adulto. Rio de Janeiro: Abril, 2012.

FREIRE P. Pedagogia da autonomia: saberes necessários à prática educativa. 41. ed. São Paulo: Paz e Terra, 2010.

FREITAS, A, L, P.; RODRIGUES, S, G. A avaliação da confiabilidade de questionário: uma análise utilizando o coeficiente de alfa de Cronbach. In: SIMPÓSIO DE ENGENHARIA DE PRODUÇÃO, 12., 2005, Bauru. Bauru: Unes, 2005.

GOMES, R. Análise e interpretação de dados de pesquisa qualitativa. In: MINAYO, M. C. S. (Org.). Pesquisa social: teoria, método e criatividade. Petrópolis: Vozes, 2010. p. 79-108. <Disponível em: <www.scielo.br/scielo.php?script=sci_ nlinks\&ref=000135\&pid=S0103...lng $>$. Acesso em: 30 jun. 2016.

GRANT, M. M. Getting a grip on project-based learning Theory, cases. Meridian: A middle School Computer Technologies Jornal, v. 5, n. 1, 2002.

HAIR, J. F. et al. Análise multivariada de dados. 5. ed. Porto Alegre: Bookman, 2005.

KNOWLES, M. S.; HOLTON III, E. F.; SWANSON, R. A. Aprendizagem de resultados: uma abordagem prática para aumentar a efetividade da educação corporativa. Rio de Janeiro: Elsevier, 2009.

LUCKESI, C. C. Avaliação da aprendizagem componente do ato pedagógico. São Paulo: Cortez, 2011.

MALCON, T. A construção do conhecimento segundo Piaget. 2012. Disponível em: $<$ www.cerebromente.org.br/n08/mente/construtivismo/construtivismo.htm>. Acesso em: 12 dez. 2016. 
MENDES, M. C. et al. Andragogia e a didática do ensino superior: novo lidar com o aprendizado do adulto em EAD. Gestão e Saúde [On-line], v. 3, n. 1, 2012. Disponível em: <periódicos.unb.br/index.php/article/view/14057>.

MORIN E. Introdução ao pensamento complexo. Porto Alegre: Sulina, 2002.

RIBEIRO, Célia. Metacognição: um apoio ao processo de aprendizagem. Psicologia: Reflexão e Crítica, v. 16, n. 1, p. 109-116, 2003.

RIBEIRO, L. R. C. A Aprendizagem Baseada em Problemas (ABP): uma implementação na educação em engenharia na voz dos atores. 2005. 209f. Tese (Doutorado em Educação) - Universidade Federal de São Carlos, São Carlos, 2005.

SMOLKA, M. L. R. M. A construção da autonomia no contexto pedagógico do estudante e o ensino das ciências da saúde: pelas veredas da educação médica. 2011. Dissertação (Mestrado) - Instituto Federal de Educaçaõ, Ciência e Tecnologia do Rio de Janeiro. Rio de Janeiro, 2011.

SOUZA, C. S.; IGLESIAS, A. G.; PAZIN FILHO, A. Estratégias inovadoras para métodos de ensino tradicionais - aspectos gerais. In: SIMPÓSIO: TÓPICOS FUNDAMENTAIS PARA A FORMAÇÃO E O DESENVOLVIMENTO DOCENTE PARA PROFESSORES DOS CURSOS DA ÁREA DA SAÚDE. Cap. VI. Medicina, Ribeirão Preto, v. 47, n. 3. 2014. Disponível em: <http://www.revistas.usp.br/rmrp/article/view/86617/89547>. Acesso em: 14 set. 2016.

TOLEDO A. C. C.; IBIAPINA C. C.; LOPES S. C. F. Aprendizagem baseada em problemas: uma nova referência para a construção do currículo médico. Revista Médica de Minas Gerais, v. 18, n. 2, p. 123-131, 2008. 